\title{
Pattern-Based Annotation of HTML-Streams
}

\author{
Florian Schmedding, Max Schwaibold, and Kai Simon \\ Institute of Computer Science, \\ Albert-Ludwigs-University Freiburg, Germany \\ \{schmeddi, schwaibo,ksimon\}@informatik.uni-freiburg.de
}

\begin{abstract}
Web pages containing RDFa markup facilitate a broad range of new agents that improve their usability for human readers. Unfortunately, there still exist only few web sites featuring such annotations. In this paper, we demonstrate Atheris, a system that annotates structured web pages by means of our web data extraction tool ViPER. Atheris runs inside a web proxy service, making it transparently available. Our approach enables the web browser - the mostly used web agent - to operate intelligently on the displayed page by providing a semantic view over previously 'meaningless' data in order to support human readers.
\end{abstract}

\section{Introduction}

Many new applications are facilitated by $R D F a$ [1, a recent W3C-recommendation. RDFa-aware agents are able to combine the visual representation of data with their meaning, thereby offering additional value to users (cf. [2] and Sec.3). In this paper, we will demonstrate our approach to annotating structured web pages to enable the application of such agents. An example is shown in Fig. 1] It depicts a product list of an online pharmacy with information about medicine (price, producer, and additional information). Here, an agent could compare products from other pages with the current products depending on the user's actual interaction with the displayed page and his current focus of interest (w.r.t. the displayed content or the mouse pointer, for instance).

\begin{tabular}{|c|l|l|l|}
\hline Image & \multicolumn{1}{|c|}{ Title } & Producer & \multicolumn{1}{c|}{ Price } \\
\hline & $\begin{array}{l}\text { Aspirin } \\
20 \text { pcs. - pills } \\
\text { article no. } 1738533\end{array}$ & Bayer & $\begin{array}{l}\text { Normal price: } 3.99 \mathrm{~S} \\
\text { Your price: } 2.99 \mathrm{~S}\end{array}$ \\
\hline
\end{tabular}

Fig. 1. Product list excerpt from an online pharmacy

Atheris, our semi-automatic web data annotation tool, recognizes such pattern structures and annotates the contained data. By implementing it into a web proxy we make the annotation step invisible to the user and provide a semantic view on previously not machine-readable web sites. 


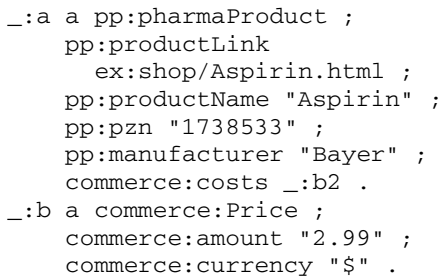

Listing 1. RDF statements for the pharmacy example

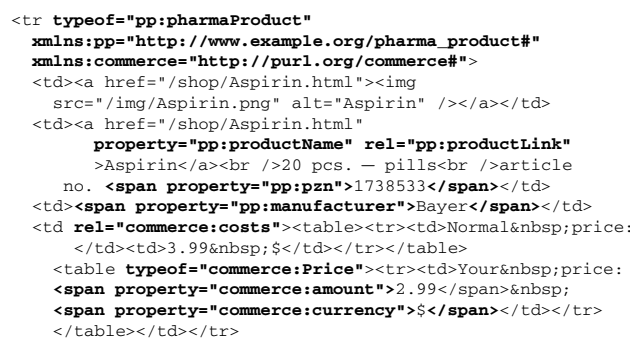

Listing 2. XHTML snippet. RDFa inserted according to Listing 1 appears in bold print.

With RDFa, arbitrary RDF statements $[3$ can be embedded into XHTML at any position using the structure and content of the document itself to express them. We refer to the reference specification in [4] instead of explaining RDFa and show a reasonable RDF description (Listing 1) and its embedding (Listing 2) for the before-mentioned example.

\section{$1.1 \quad$ Related Work}

Research in the field of web data extraction and annotation has brought up a broad range of approaches and tools (cf. the surveys in [5] and [6]). Some of these systems are specialized in extracting relevant data from pages (e.g. Lixto [7, SparqPlug [8]), but w.r.t. RDFa-agents it is important to include the semantic annotations into the page. For instance, Piggy Bank [9] uses extractions and is thus unable to adapt the offered functionality to the user's current interest. Manual tools like Annoted 1 do not fit the requirements either although Annotea relates the annotations with corresponding page elements by XPointer expressions. MnM [10] or S-CREAM [1] are better suited. Both are able to add additional XML-tags around the relevant data but further knowledge is necessary to convert them into RDF. Similar to PowerMagpie2, they are rather designed for continuous text than for structured content. Thresher [12] is most related to our approach yet uses a non-standardized formalism to relate terms and concepts within documents. Other systems like Greasemonkey ${ }^{3}$ and Chickenfoot [13] are also adding further functionality to page elements identified by screenscraping techniques. However, this is achieved by hard-coded scripts within the document.

In contrast, our system uses RDFa to annotate the identified concepts and relations between them. Hence, its output can be used by existing RDFa-agents and so is independent from the annotation process. Additionally, Atheris is available as proxy service to provide an easy access for web browsers.

\footnotetext{
1 http://www.w3.org/2001/Annotea/

2 http://powermagpie.open.ac.uk/

3 http://greasemonkey.mozdev.org/
} 


\section{$1.2 \quad$ ViPER}

Our wrapper extraction tool ViPER [14 automatically identifies and extracts different regularly structured content with high accuracy according to several visual features. We build Atheris upon it due to its fast and robust pattern matching algorithm. ViPER was refined into RexViPER [15] to also derive postprocessing rules from the extracted and aligned data. The purpose of these rules is the automatic labeling of named entities, the splitting of strings, and the removal of text from data entities to simplify further rule assignments. Rules can be assigned manually or with a drag-and-drop tool for non-expert users as implemented in [16]. The generated extraction pattern matching the structure of the data records in our example is described by the (abbreviated) HTML tag sequence:

\begin{tabular}{|l||c|c|c|c|c|c|c|c|c|c|c|c|c|c|c|c|c|}
\hline Position & 0 & 1 & 2 & 3 & 4 & 5 & 6 & 7 & 8 & 9 & 10 & 11 & 12 & 13 & 14 & $\ldots$ & 37 \\
\hline Pattern & $\langle\mathrm{TR}\rangle$ & $\langle\mathrm{TD}\rangle$ & $\langle\mathrm{A}\rangle$ & $\langle\mathrm{IMG}\rangle$ & $\langle/ \mathrm{A}\rangle$ & $\langle/ \mathrm{TD}\rangle$ & $\langle\mathrm{TD}\rangle$ & $\langle\mathrm{A}\rangle$ & $\mathrm{TEXT}$ & $\langle/ \mathrm{A}\rangle$ & $\langle\mathrm{BR}\rangle$ & $\mathrm{TEXT}$ & $\langle\mathrm{BR}\rangle$ & $\mathrm{TEXT}$ & $\langle/ \mathrm{TD}\rangle$ & $\ldots$ & $\langle/ \mathrm{TR}\rangle$ \\
\hline
\end{tabular}

The attached set of rules (depicted in Listing 3) consists of both automatically learned rules found with the aid of RexViPER and manually assigned RDFa annotation rules to enrich the appropriate data items with RDFa semantics. The first argument of a rule references a pattern element by its position. For instance, the rule split(32,\&nbsp;) splits the content of the 32nd element according to hard space. Here, the content will be split into Your, price: 2.99 , and $\$$. The resulting substrings can be referenced using the sub-indices 32.0, 32.1 and 32.2. RDFa-specific rules are shown in the next section.

\section{Atheris}

To annotate pages, Atheris has to extend their structure to make the pattern's data 'visible' to agents instead of disregarding it. Thus, being an extraction tool, some modifications inside ViPER were necessary to facilitate annotations. The changes are summarized by three points. First, to insert the RDFa-markup correctly (mostly attributes and span-elements), we store all tag positions w.r.t. the HTML-stream. Second, we implement new rules besides adapting the existing ones to the stored tag positions. Third, to ensure the well-formedness of the output, changes to the handling of formatting elements like $b$ and $i$ are necessary.

Annotation Rules. Listing 3 represents the rules used to annotate the example page. Additionally, two rules are used to set up the namespaces in the tr-element. We illustrate the respective results with Listing 2 The rules typeof and relation insert @typeof into elements 0 and 29 respectively @rel into elements 7 and 18. This suffices to express triples 1, 2, 6, and 7 in Listing 1 . The remaining statements follow from property which delimits the desired literals by span-tags (except for element 7) and inserts the associated predicate into the @property-attribute. Rules to declare datatypes or other constructs are not used here and therefore left out. We want to highlight the join-rule that combines separated text elements into a single literal: In the example, this could be used to attach elements 11 and 13 to a predicate like pp:additionalInformation. 


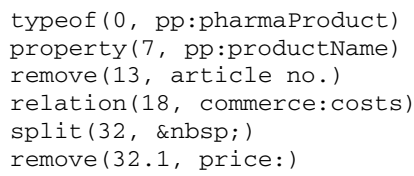

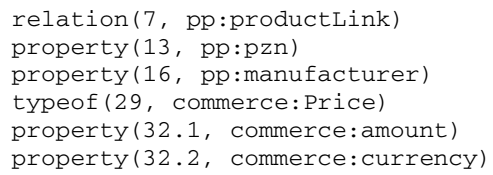

Listing 3. Annotation rules

Two or More Patterns. When a web page contains more than one type of structured information, it is not known in advance which kind of data is going to be used by agents in the annotation scenario. It is therefore necessary to annotate all patterns before sending the page to the client. We enhanced the pattern recognition engine in ViPER to execute concurrent parallel pattern matching threads in spite of running Atheris for each pattern serially. This approach benefits from a better performance by avoiding multiple input parsing.

\subsection{Proxy}

To deliver the annotated pages easily to web browsers, we decided to implement an HTTP proxy service due to the transparent access. With the proxy, users also benefit from sharing the annotation patterns and rules. Incoming requests are classified as either annotable or others. Not annotable requests are handled by the proxy $y^{4}$ itself while annotable requests are passed over to Atheris and processed according to the proxy's pattern and rule database.

Like ViPER, Atheris is working on the raw HTML stream in favor of a smaller latency for the clients. This streaming capability is the reason why it is possible to use chunked encoding for the annotation handling giving better response times and thus making the proxy as transparent as possible for clients.

\section{Conclusion and Future Work}

We have presented Atheris, our system to annotate web pages with RDFamarkup. Atheris relies on the robust pattern-matching technique of ViPER and introduces an abstraction layer between web pages and additional functionality for these pages (changing the appearance, adding information, etc.) by embedding meta data with RDFa. Due to the provided semantic view over previously not machine-readable data, the web browser can be turned into a semantic user agent operating on structured pages in a meaningful way that has been impossible before. Because of the clear separation between data and functionality it is also possible to change either of the components without side effects.

Despite its just mentioned advantages, an HTTP proxy is unable to annotate streams in secure channels (cf. also [13]). It has also limited resources and can quickly induce a bottleneck under heavy load. Therefore, and because the primary use of annotated pages would occur in combination with the rendered presentation, it is an option to implement the system directly inside a browser. If the annotation step could be performed directly inside the browser's parser,

\footnotetext{
${ }^{4}$ Subclass of Mentalis proxy, http://www.mentalis.org/soft/projects/proxy
} 
this would even improve the performance. Of course, to keep the benefit of collaboration, the system should request the patterns and rules from a common store. To make it available to other RDFa-consuming systems it could be published as web service, too. We are also improving the annotation rules to capture more cases. For instance, the rule to annotate currencies should implement an automatic conversion from symbols to the required ISO-4217 literals (\$ to USD in our example) for commerce: currency. Additionally, we are currently working on a browser plugin that integrates RDFa into the DOM of web pages to identify the annotated parts of a page with SPARQL queries. This enables the plugin to show feedback from other agents just beside the annotations.

\section{References}

1. Adida, B., Birbeck, M.: RDFa Primer (2008), http://www.w3.org/TR/2008/NOTE-xhtml-rdfa-primer-20081014/

2. Adida, B., Hausenblas, M.: RDFa Use Cases (2007), http://www.w3.org/TR/2007/WD-xhtml-rdfa-scenarios-20070330/

3. Manola, F., Miller, E.: RDF Primer (2004), http://www.w3.org/TR/2004/REC-rdf-primer-20040210/

4. Adida, B., Birbeck, M., McCarron, S., Pemberton, S.: RDFa in XHTML: Syntax and Processing (2008), http://www.w3.org/TR/2008/REC-rdfa-syntax-20081014

5. Chang, C.H., Kayed, M., Girgis, M.R., Shaalan, K.F.: A Survey of Web Information Extraction Systems. IEEE TKDE 18(10), 1411-1428 (2006)

6. Uren, V., Cimiano, P., Iria, J., Handschuh, S., Vargas-Vera, M., Motta, E., Ciravegna, F.: Semantic Annotation for Knowledge Management: Requirements and a Survey of the State of the Art. Web Semantics (4), 14-28 (2006)

7. Baumgartner, R., Flesca, S., Gottlob, G.: Visual Web Information Extraction with Lixto. In: VLDB, San Francisco, CA, USA, pp. 119-128 (2001)

8. Coetzee, P., Heath, T., Motta, E.: SparqPlug: Generating Linked Data from Legacy HTML, SPARQL and the DOM. In: LDOW, Bejing, China (2008)

9. Huynh, D., Mazzocchi, S., Karger, D.: Piggy Bank: Experience the Semantic Web inside your Web Browser. Web Semant 5(1), 16-27 (2007)

10. Vargas-Vera, M., Motta, E., Domingue, J., Lanzoni, M., Stutt, A., Ciravegna, F.: MnM: Ontology Driven Semi-Automatic and Automatic Support for Semantic Markup. In: Gómez-Pérez, A., Benjamins, V.R. (eds.) EKAW 2002. LNCS, vol. 2473, pp. 379-391. Springer, Heidelberg (2002)

11. Handschuh, S., Staab, S., Ciravegna, F.: S-CREAM - Semi-automatic CREAtion of Metadata. In: Gómez-Pérez, A., Benjamins, V.R. (eds.) EKAW 2002. LNCS, vol. 2473, pp. 358-372. Springer, Heidelberg (2002)

12. Hogue, A., Karger, D.: Thresher: Automating the unwrapping of semantic content from the world wide web. In: WWW, Chiba, Japan, pp. 86-95 (2005)

13. Bolin, M., Webber, M., Rha, P., Wilson, T., Miller, R.C.: Automation and Customiztion of Rendered Web Pages. In: UIST, Seattle, WA, USA, pp. 163-172 (2005)

14. Simon, K., Lausen, G.: ViPER: Augmenting Automatic Information Extraction with Visual Perceptions. In: CIKM, Bremen, Germany, pp. 381-388 (2005)

15. Simon, K., Hornung, T., Lausen, G.: Learning Rules to Pre-process Web Data for Automatic Integration. In: RuleML, Athens, GA, USA, pp. 107-116 (2006)

16. Hornung, T., Simon, K., Lausen, G.: Mashing Up the DEEP Web - Research in Progress. In: WEBIST (2), Madeira, Portugal, pp. 58-66 (2008) 